\title{
Carbon and Nitrogen Utilization by Micromonospora Strains
}

\author{
Isao KaWAmoto, Tetsuo OKa and Takashi NARA \\ Tokyo Research Laboratory, Kyowa Hakko Co., \\ 3-6-6 Asahimachi, Machidashi, Tokyo 194, Japan
}

Received January 8, 1982

\begin{abstract}
The utilization of 28 nitrogen compounds and 24 carbohydrates by 23 strains of Micromonospora was examined after growth on a chemically defined medium. Inorganic ammonium salts served as better nitrogen sources than nitrate salts, and most of the organisms metabolized acidic or basic amino acids more extensively than neutral ones, except for L-serine. Utilization of carbohydrates in the defined medium did not always correspond to that observed when Luedemann's medium was used as a basal medium. Experiments using $p$-nitrophenylglycosides indicated that $\alpha$-glucosidase, $\beta$-glucosidase, $\beta$-galactosidase and $\beta$ - $N$-acetylglucosaminidase were present in all the strains. The diagnostic properties found in this study are discussed in terms of the classification of Micromonospora species.
\end{abstract}

Relatively little is known about the physiological and biochemical properties of Micromonospora strains, even though these same strains produce numerous antibiotics. ${ }^{1,2)}$ Very recently, we indicated that these organisms had a new type of peptidoglycan ${ }^{3)}$ and bore spores more resistant to heat and to polar solvents than those of Streptomyces strains. ${ }^{4)}$ The utilization pattern of carbohydrates has been used extensively to characterize the species of Micromonospora, as well as those of Streptomyces. A chemically defined basal medium for carbohydrate utilization, such as the inorganic salts medium devised by Pridham and Gottlieb, ${ }^{5}$ ) has been used to identify Streptomyces strains. On the other hand, the $0.5 \%$ yeast extract medium recommended by Luedemann and Brodsky ${ }^{6}$ has been employed in the determination of many strains of Micromonospora, since they are unable to maintain sufficient growth on inorganic nitrogen media. However, the results obtained using the Luedemann's medium were inaccurate when carbohydrates were poorly utilized or not utilized at all, since the organisms can still grow on the medium in the absence of carbohydrate. This study was undertaken to clarify the utilization pattern of carbohydrates by Micromonospora strains.

\section{MATERIALS AND METHODS}

Organisms. Twenty three strains were used in this study. Three strains, Micromonospora inositola, M. sagamiensis and $M$. olivasterospora were isolated in our laboratory from soils. Their cultures have been deposited at the American Type Culture Collection, Rockyville, Maryland, U.S.A., under accession numbers ATCC 31010, 21803 and 21819, respectively. Other strains were obtained from American Type Culture Collection (ATCC); Kaken Chemical Co. Ltd. (KCC), Tokyo, Japan; Institute for Fermentation (IFO), Osaka, Japan and Northern Regional Research Laboratory (NRRL), Peoria, Illinois, U.S.A.

Preparation of inocula. Each organism was inoculated into $10 \mathrm{ml}$ of a seed medium in a $50 \mathrm{ml}$ tube and incubated for $2 \sim 3$ days on a reciprocating shaker. The seed medium (SR) consisted of $2 \%$ glucose (Nakarai Chemical Co.), $0.2 \%$ beef extract (Kyokuto Seiyaku Co.), $0.2 \%$ yeast extract (Daigo Eiyo Chemical Co.), $0.2 \%$ Polypepton (Daigo Eiyo Chemical Co.) and $0.1 \% \mathrm{CaCO}_{3}$ (Kanto Chemical Co.). The pH was adjusted to 7.3 before autoclaving. The culture was harvested by centrifugation and washed three times with sterile saline water. The mycelial suspension was prepared by adding an equal volume of sterile saline water to the packed mycelia after centrifugation.

Growth rate at different $p H$ 's. An autoclaved SR medium was adjusted to various $\mathrm{pH}$ values $(4.5 \sim 9.5)$ with a sterile $\mathrm{HCl}$ or $\mathrm{NaOH}$ solution. Enough of the organism was inoculated into the medium to give an optical density of 0.2 at $660 \mathrm{~nm}$, and a $10 \mathrm{ml}$ aliquot of the medium was 
removed aseptically into a sterile $50 \mathrm{ml}$ test tube. The culture was then incubated for $8 \mathrm{hr}$ at $28^{\circ} \mathrm{C}$ on a reciprocating shaker.

Effect of phosphate on growth. Mycelial suspensions $(0.1 \mathrm{ml})$ were inoculated into SR media containing different concentrations of phosphate, and then incubated overnight at $28^{\circ} \mathrm{C}$ on a reciprocating shaker.

Nitrogen and carbon utilization. The basal mineral medium consisted of $10 \mathrm{~mm} \mathrm{KH}_{2} \mathrm{PO}_{4}, 5 \mathrm{~mm} \mathrm{MgSO}_{4}, 10 \mu \mathrm{M}$ $\mathrm{FeSO}_{4}, 10 \mu \mathrm{M} \mathrm{ZnSO}_{4}, 10 \mu \mathrm{M} \mathrm{CuSO}_{4}, 1 \mu \mathrm{M} \mathrm{MnSO}_{4}$ and $10 \mathrm{~mm} \mathrm{CaCO}_{3}$. The medium was supplemented with cysteine $(0.1 \mathrm{mg} /$ liter $)$ and vitamins: thiamine $\cdot \mathrm{HCl}(0.2 \mathrm{mg}$ / liter $)$, riboflavin $(0.2 \mathrm{mg} /$ liter $)$, pyridoxine $\cdot \mathrm{HCl}(0.2 \mathrm{mg} /$ liter $)$ cyanocobalamin $(0.2 \mathrm{mg} /$ liter $)$, nicotinic acid $(0.5 \mathrm{mg} /$ liter $)$, calcium pantothenate $(0.05 \mathrm{mg} /$ liter $)$, folic acid $(0.05 \mathrm{mg} /$ liter $), p$-aminobenzoic acid $(0.05 \mathrm{mg} /$ liter $)$, biotin $(0.1 \mathrm{mg} /$ liter $)$ and inositol $(0.1 \mathrm{mg} /$ liter $)$. The agar medium was prepared by adding $1.5 \%$ purified agar (Difco). Each nitrogen source was added aseptically from a concentrated solution to the basal medium, supplemented with $100 \mathrm{~mm}$ glucose, to give a final concentration of $50 \mathrm{~mm}$ as nitrogen. Two kinds of basal media were used for carbohydrate utilization: a defined medium supplemented with $25 \mathrm{~mm} \mathrm{NH}_{4} \mathrm{NO}_{3}$, and Leudemann's medium $(0.5 \%$ yeast extract and $\left.0.1 \% \mathrm{CaCO}_{3}\right){ }^{6}{ }^{6}$ With the exception of soluble starch and salicin, each carbon source was sterilized by filtration and added aseptically to a melted basal agar medium, to yield a final concentration of $1 \%$. Soluble starch or salicin was autoclaved separately, and then added. A loopful of a mycelial suspension was streaked over the surface of the agar medium and incubated at $28^{\circ} \mathrm{C}$ for 14 days, while $0.1 \mathrm{ml}$ of a mycelial suspension was inoculated into a liquid medium and incubated for 6 days at $28^{\circ} \mathrm{C}$ on a reciprocating shaker.

Measurement of growth. Growth on an agar medium was estimated visually, whereas that in a liquid medium was measured as optical density at $660 \mathrm{~nm}$ after the residual $\mathrm{CaCO}_{3}$ was dissolved by adding $0.5 \mathrm{ml}$ of $2 \mathrm{~N} \mathrm{HCl}$ per $9.5 \mathrm{ml}$ of the culture broth.

Detection of glycosidase activities. The underlying principle of this test was that applied previously to the characterization of Enterobacteriaceae. ${ }^{7)}$ The mycelial suspension was treated with a sonic oscillator for $5 \mathrm{~min}$ in an ice bath. The reaction mixture consisted of $0.1 \mathrm{ml}$ of the sonicate and $0.9 \mathrm{ml}$ of each $p$-nitrophenylglycoside solution (Nakarai Chemical Co., $1 \mathrm{mg}$ per $\mathrm{ml}$ of $1 / 15 \mathrm{M}$

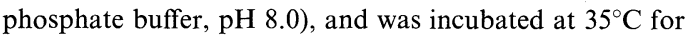
$4 \mathrm{hr}$. The activities of the glycosidases were estimated visually by formation of a yellow color because of released nitrophenol.

\section{RESULTS}

\section{Growth conditions}

Since Micromonospora olivasterospora ATCC 21819 grew dispersively in submerged culture, the growth rate was determined by measuring optical density in liquid media adjusted to different pH's (Fig. 1). The optimal $\mathrm{pH}$ for growth of the strain was approximately 7.5 , at which value the doubling time was nearly $4.5 \mathrm{hr}$. At pH values below 5.0 or above 9.5, growth was not observed, and a lag time was required for growth at $\mathrm{pH} 5.3$ or $\mathrm{pH} 8.9$. At these values the doubling time was $8.3 \mathrm{hr}$ or $6.5 \mathrm{hr}$, respectively. Similar experiments were undertaken for 6 other strains: $M$. chalcea ATCC 12452, M. echinospora subsp. pallida NRRL 2996, M. globosa KCC A-0126, $M$. grisea NRRL 3800, $M$. inyoensis NRRL 3292 and $M$. parva KCC A-0127. Almost the same tendencies were demonstrated with these strains as were observed with $M$. olivasterospora, and their doubling times at $\mathrm{pH} 7.0$ ranged from $4.5 \mathrm{hr}$ to $7 \mathrm{hr} 5 \mathrm{~min}$. Among the

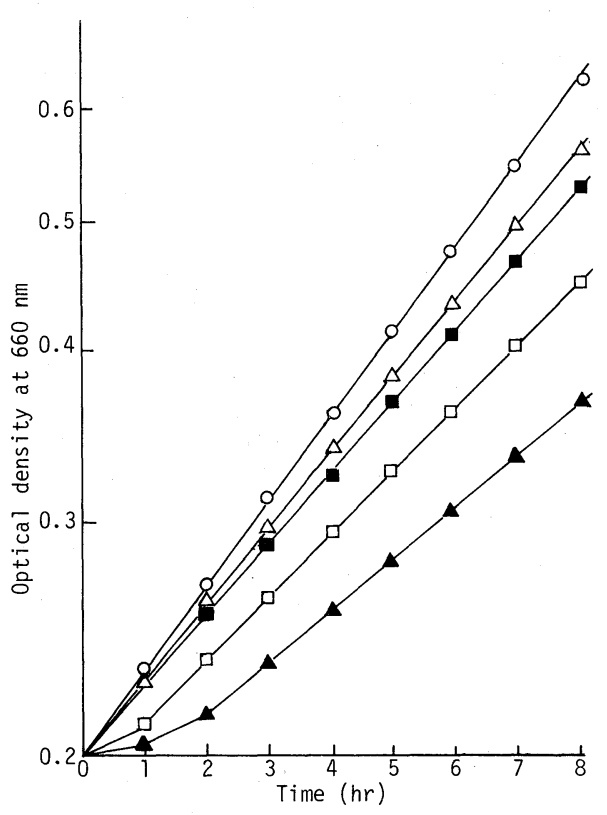

FIG. 1. Growth of Micromonospora olivasterospora ATCC 21819 in Media at Various pH's.

O, $\mathrm{pH} 7.5 ; \triangle, \mathrm{pH} 7.0$ and $\mathrm{pH} 7.9 ; \square, \mathrm{pH} 5.9 ; \square, \mathrm{pH} 8.9$;

$\Delta, \mathrm{pH} 5.3$. 


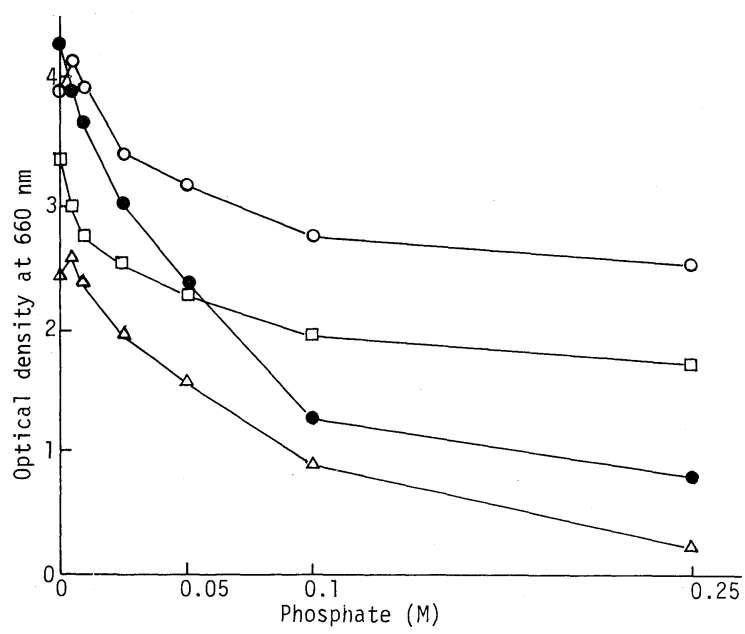

FIG. 2. Effect of Phosphate on Growth of Micromonospora Strains.

O, M. chalcea ATCC 12452; $\square$, M. globosa KCC A-0126; ATCC 21819.

strains tested, M. chalcea ATCC 12452 had the best growth rate.

In an attempt to examine the effect of phosphate concentration on growth, four strains were cultivated in media with various concentrations of phosphate. As shown in Fig. 2, a significant decrease of mycelial yield occurred at concentrations higher than $0.01 \mathrm{M}$ phosphate. This effect was most remarkable with M. parva KCC A-0127. Therefore, $0.01 \mathrm{M}$ was set as the final phosphate concentration in the chemically defined medium.

When the growth of each of the 23 strains was tested on a chemically defined agar medium containing $100 \mathrm{~mm}$ glucose, $25 \mathrm{~mm} \mathrm{~L}$ asparagine and mineral salts, $M$. narashino KCC A-0129 and M. zionensis NRRL 5466 did not grow at all, and some strains grew only slightly. However, the addition of supplements of cysteine and vitamins, as described in Materials AND Methods, allowed sufficient growth. Consequently, a chemically defined medium was devised, which could be applied to determination of nitrogen or carbon utilization by all the strains examined.

\section{Utilization of nitrogen sources}

Approximately half of the strains tested showed poor or no growth in a liquid medium containing sodium nitrate or potassium nitrate as the nitrogen source; whereas almost all the strains exhibited moderate or abundant growth in media containing ammonium salts (Table I). When the strains which utilized nitrate well as the sole nitrogen source were cultivated in a medium containing ammonium nitrate, their culture broths became acidic, a result similar to that observed when other media containing ammonium salts had been used. However, media having nitrate salts as the nitrogen source yielded alkaline culture broths. Thus, ammonium-nitrogen appeared to be a more favorable nitrogen source than nitrate-nitrogen for the growth of Micromonospora strains. In the medium containing ammonium salts, those cultures which grew moderately well eventually lowered the $\mathrm{pH}$ below 5.0, at which point they could not grow any longer. They were unable to achieve abundant growth because of the rapid fall of the $\mathrm{pH}$ from an increased residual anion concentration after consumption of the ammonium ion.

In experiments using 20 amino acids as sole nitrogen sources, neither L-phenylalanine nor L-tyrosine permitted growth of all the strains used, while L-leucine, L-isoleucine and Lmethionine allowed only poor growth of a few 
Table I. Utilization of Inorganic Nitrogen Compounds by Micromonospora Strains in a Defined Medium

\begin{tabular}{|c|c|c|c|c|c|c|c|c|c|c|c|c|c|}
\hline \multirow[t]{2}{*}{ Micromonospora strains } & & \multicolumn{2}{|c|}{$\begin{array}{l}\mathrm{NaNO}_{3} \\
(50 \mathrm{~mm})\end{array}$} & \multicolumn{2}{|c|}{$\begin{array}{c}\mathrm{KNO}_{3} \\
(50 \mathrm{~mm})\end{array}$} & \multicolumn{2}{|c|}{$\begin{array}{c}\mathrm{NH}_{4} \mathrm{NO}_{3} \\
(25 \mathrm{~mm})\end{array}$} & \multicolumn{2}{|c|}{$\begin{array}{c}\left(\mathrm{NH}_{4}\right)_{2} \mathrm{SO}_{4} \\
(25 \mathrm{~mm})\end{array}$} & \multicolumn{2}{|c|}{$\begin{array}{c}\left(\mathrm{NH}_{4}\right)_{2} \mathrm{HPO}_{4} \\
(25 \mathrm{~mm})\end{array}$} & \multicolumn{2}{|c|}{$\begin{array}{c}\mathrm{NH}_{4} \mathrm{Cl} \\
(50 \mathrm{~mm})\end{array}$} \\
\hline & & G & $\mathrm{pH}$ & G & $\mathrm{pH}$ & G & $\mathrm{pH}$ & G & $\mathrm{pH}$ & G & $\mathrm{pH}$ & G & $\mathrm{pH}$ \\
\hline M. purpurea & NRRL 2953 & ++ & 8.5 & ++ & 8.5 & ++ & 5.6 & ++ & 5.8 & ++ & 5.5 & ++ & 5.6 \\
\hline M. echinospora subsp. ferruginea & NRRL 2995 & ++ & 8.6 & ++ & 8.5 & ++ & 5.6 & ++ & 5.5 & ++ & 5.5 & ++ & 5.6 \\
\hline M. echinospora subsp. pallida & NRRL 2996 & ++ & 8.0 & ++ & 8.0 & ++ & 5.4 & ++ & 5.6 & ++ & 4.3 & ++ & 5.4 \\
\hline M. echinospora & NRRL 2985 & ++ & 7.8 & + & 7.2 & ++ & 5.4 & ++ & 5.7 & ++ & 5.3 & ++ & 5.5 \\
\hline$M$. inyoensis & NRRL 3292 & ++ & 8.0 & ++ & 9.0 & + & 5.9 & + & 5.7 & ++ & 5.3 & + & 5.6 \\
\hline$M$. parva & KCC A-0127 & ++ & 6.7 & ++ & 6.9 & + & 4.1 & + & 4.2 & ++ & 4.5 & + & 4.1 \\
\hline M. grisea & NRRL 3800 & + & 8.4 & + & 8.3 & ++ & 5.0 & ++ & 5.1 & ++ & 5.0 & ++ & 5.1 \\
\hline M. chalcea & ATCC 12452 & + & 8.0 & + & 8.0 & ++ & 5.2 & ++ & 5.2 & ++ & 5.3 & ++ & 5.2 \\
\hline M. inositola & ATCC 31010 & + & 7.9 & + & 8.0 & ++ & 4.2 & + & 4.4 & ++ & 4.2 & + & 4.3 \\
\hline M. globosa & KCC A-0126 & + & 7.9 & + & 7.9 & + & 4.7 & + & 4.7 & + & 5.6 & + & 4.8 \\
\hline M. halophytica subsp. nigra & NRRL 3097 & + & 7.8 & \pm & 7.8 & + & 4.2 & + & 4.2 & + & 4.3 & \pm & 5.6 \\
\hline M. zionensis & NRRL 5466 & \pm & 7.6 & \pm & 7.7 & ++ & 5.4 & ++ & 5.5 & ++ & 5.5 & ++ & 5.4 \\
\hline M. olivasterospora & ATCC 21819 & \pm & 7.5 & $\overline{ \pm}$ & 7.5 & ++ & 6.0 & ++ & 5.4 & + & 5.4 & ++ & 5.7 \\
\hline M. melanosporea & IFO 12515 & \pm & 7.6 & - & 7.5 & ++ & 5.8 & ++ & 5.8 & + & 6.5 & ++ & 5.7 \\
\hline M. carbonacea subsp. aurantica & NRRL 2997 & \pm & 7.7 & \pm & 7.9 & + & 5.7 & + & 4.8 & ++ & 5.1 & + & 4.8 \\
\hline M. carbonacea & NRRL 2972 & \pm & 7.8 & \pm & 7.9 & + & 4.7 & + & 4.7 & + & 5.6 & + & 4.9 \\
\hline M. purpureochromogenes & ATCC 27007 & \pm & 8.4 & \pm & 8.4 & + & 4.3 & + & 4.5 & + & 4.4 & + & 4.3 \\
\hline M. rosaria & NRRL 3718 & \pm & 7.9 & \pm & 7.9 & + & 6.2 & \pm & 6.8 & + & 6.3 & + & 6.0 \\
\hline M. megalomicea subsp. nigra & NRRL 3275 & - & 7.0 & - & 7.0 & + & 5.3 & + & 5.2 & + & 5.6 & + & 5.3 \\
\hline M. sagamiensis & ATCC 21803 & - & 7.0 & - & 7.0 & + & 6.0 & \pm & 5.7 & + & 5.7 & \pm & 5.9 \\
\hline M. narashino & KCC A-0129 & - & 7.0 & - & 7.0 & ++ & 4.3 & ++ & 4.3 & ++ & 6.1 & ++ & 4.7 \\
\hline M. fusca & NRRL B-943 & - & 7.0 & - & 7.0 & ++ & 5.6 & ++ & 5.8 & ++ & 5.6 & ++ & 5.6 \\
\hline
\end{tabular}

G: Growth after 6 days of incubation at $28^{\circ} \mathrm{C}(++$, abundant; + , moderate; \pm , poor;,- no growth $) . \mathrm{pH}$ : Final $\mathrm{pH}$ values of growth cultures. 
Table II. Utilization of Amino Acids as Sole Nitrogen Sources by Micromonospora Strains in a Defined Medium

\begin{tabular}{|c|c|c|c|c|c|c|c|c|c|c|c|c|c|c|c|c|c|c|}
\hline \multirow[b]{2}{*}{ Micromonospora strains } & & \multicolumn{17}{|c|}{ Growth $^{a}$} \\
\hline & & 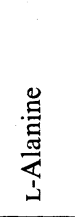 & 莺 & 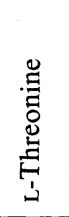 & 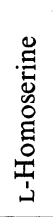 & 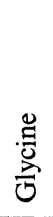 & $\underset{\frac{\pi}{\pi}}{\stackrel{\Xi}{\pi}}$ & 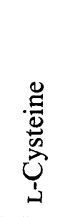 & $\frac{.}{\frac{\Xi}{0}}$ & 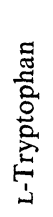 & 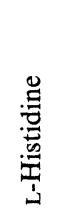 & 壳 & & 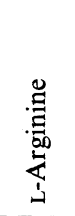 & 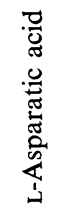 & 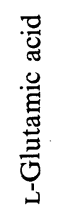 & 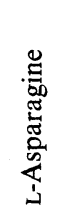 & 志 \\
\hline M. echinospora & NRRL 2985 & ++ & ++ & ++ & \pm & - & - & - & - & - & + & $+t$ & \pm & ++ & ++ & ++ & ++ & ++ \\
\hline M. echinospora subsp. pallida & NRRL 2996 & ++ & ++ & + & \pm & + & \pm & - & - & - & ++ & + & \pm & ++ & ++ & ++ & ++ & + \\
\hline M. parva & KCC A-0127 & ++ & ++ & + & - & \pm & \pm & - & \pm & - & - & \pm & $\bar{t}$ & ++ & ++ & ++ & ++ & + \\
\hline M. globosa & KCC A-0126 & ++ & ++ & + & - & \pm & - & ++ & - & - & ++ & + & + & ++ & ++ & ++ & ++ & + \\
\hline M. chalcea & ATCC 12452 & ++ & ++ & + & - & $\bar{t}$ & \pm & + & \pm & + & $+t$ & - & + & ++ & ++ & ++ & ++ & $+t$ \\
\hline M. fusca & NRRL B-943 & ++ & ++ & + & - & - & $\bar{t}$ & + & $\bar{t}$ & - & ++ & \pm & + & ++ & ++ & ++ & ++ & ++ \\
\hline M. melanosporea & IFO 12515 & ++ & ++ & \pm & - & - & \pm & \pm & - & - & ++ & - & + & ++ & ++ & ++ & ++ & ++ \\
\hline M. inositola & ATCC 31010 & $+t$ & ++ & \pm & - & - & - & $\bar{t}$ & + & - & ++ & + & + & ++ & ++ & ++ & ++ & + \\
\hline M. rosaria & NRRL 3718 & + & ++ & ++ & - & - & - & \pm & \pm & - & + & + & + & ++ & ++ & ++ & ++ & ++ \\
\hline M. purpurea & NRRL 2953 & + & ++ & + & \pm & \pm & - & + & - & - & ++ & + & \pm & ++ & ++ & ++ & ++ & + \\
\hline M. zionensis & NRRL 5466 & + & ++ & \pm & - & - & - & - & - & - & + & - & - & ++ & ++ & + & ++ & + \\
\hline M. purpureochromogenes & ATCC 27007 & + & ++ & \pm & - & - & - & - & - & - & - & \pm & \pm & ++ & ++ & ++ & ++ & + \\
\hline M. narashino & KCC A-0129 & + & + & $\bar{t}$ & - & - & \pm & + & - & - & + & $\bar{t}$ & $\overline{+}$ & ++ & ++ & ++ & ++ & + \\
\hline M. coerulea & ATCC 27008 & + & + & \pm & - & - & - & - & - & - & + & + & - & ++ & ++ & ++ & ++ & ++ \\
\hline M. carbonacea subsp. aurantica & NRRL 2997 & \pm & ++ & + & \pm & \pm & - & \pm & - & - & + & + & + & ++ & ++ & ++ & ++ & ++ \\
\hline M. echinospora subsp. ferruginea & NRRL 2995 & \pm & ++ & + & \pm & \pm & - & $\bar{t}$ & - & - & ++ & ++ & \pm & ++ & ++ & ++ & ++ & ++ \\
\hline M. grisea & NRRL 3800 & \pm & ++ & \pm & - & + & - & - & \pm & - & + & - & \pm & ++ & ++ & + & ++ & + \\
\hline M. sagamiensis & ATCC 21803 & $\stackrel{-}{-}$ & + & + & \pm & \pm & - & - & 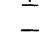 & - & + & \pm & + & + & ++ & ++ & ++ & $+t$ \\
\hline M. halophytica subsp. nigra & NRRL 3097 & - & ++ & + & - & \pm & - & + & - & - & + & \pm & \pm & + & ++ & \pm & + & + \\
\hline M. carbonacea & NRRL 2972 & - & ++ & + & - & \pm & - & + & - & + & + & $\bar{t}$ & $\bar{t}$ & ++ & ++ & ++ & ++ & $+t$ \\
\hline M. olivasterospora & ATCC 21819 & - & ++ & + & - & \pm & - & \pm & \pm & - & ++ & \pm & \pm & ++ & $+t$ & ++ & + & + \\
\hline M. megalomicea subsp. nigra & NRRL 3275 & - & + & - & - & - & - & - & $\overline{-}$ & - & \pm & \pm & \pm & + & ++ & ++ & + & + \\
\hline$M$. inyoensis & NRRL 3292 & - & \pm & + & - & - & - & - & - & - & + & \pm & \pm & ++ & ++ & ++ & ++ & + \\
\hline
\end{tabular}

a Growth after incubation for 2 weeks at $28^{\circ} \mathrm{C}(++$, abundant; + , moderate; \pm , poor; - , no growth). 


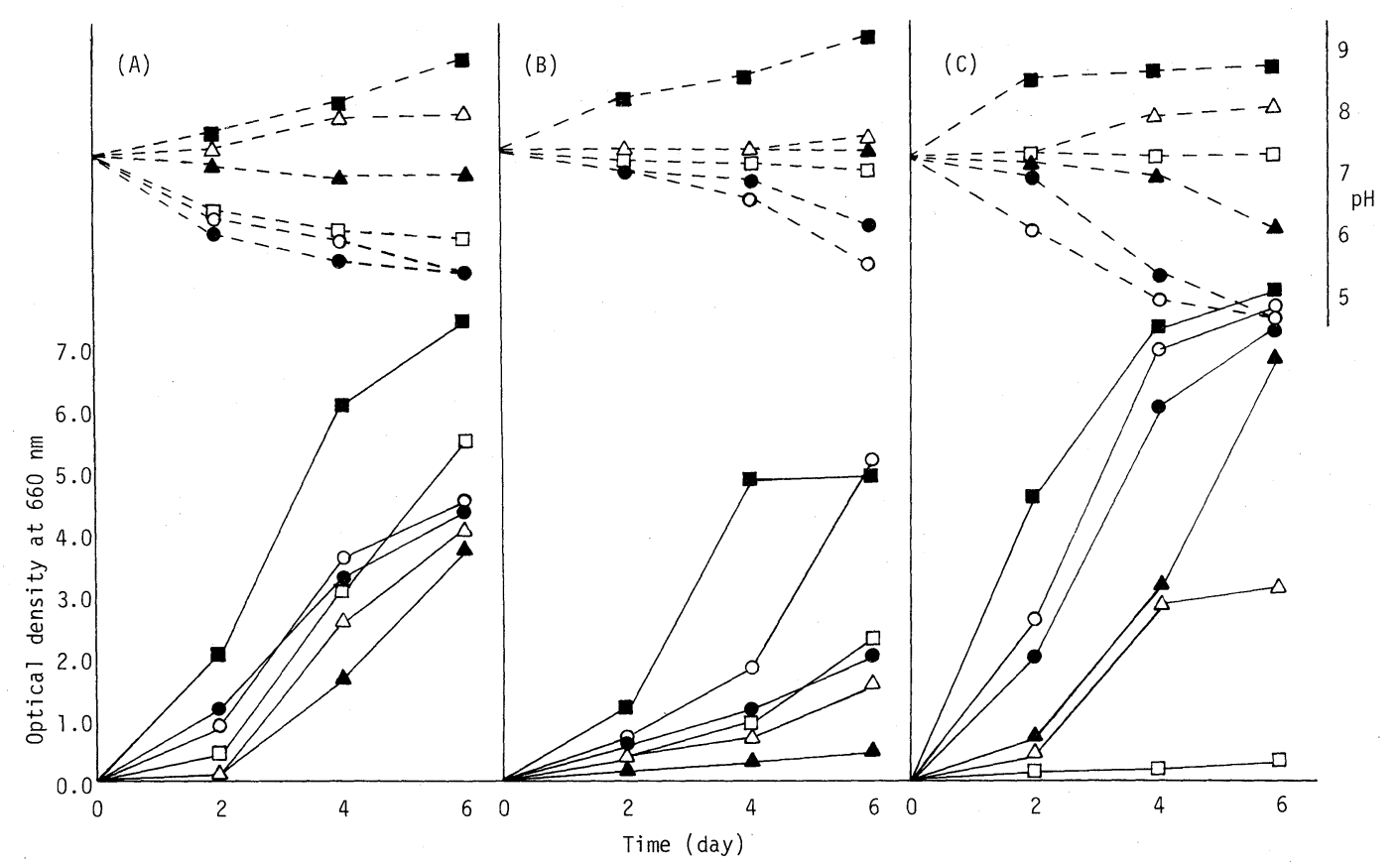

FIG. 3. Growth Curves of Micromonospora Strains in Defined Media Containing Various Nitrogen Sources. (A), Micromonospora echinospora NRRL 2953; (B), Micromonospora olivasterospora ATCC 21819; (C), Micromonospora chalcea ATCC 12452; $\mathrm{O},\left(\mathrm{NH}_{4}\right) \mathrm{SO}_{4}(25 \mathrm{mM}) ; \boldsymbol{\bullet}, \mathrm{NH}_{4} \mathrm{NO}_{3}(25 \mathrm{~mm}) ; \triangle, \mathrm{NaNO}_{3}(50 \mathrm{~mm}) ; \boldsymbol{\Delta}$, L-alanine (50 mM); $\square$, L-lysine $(25 \mathrm{~mm}) ; \square$, L-glutamic acid $(50 \mathrm{~mm}) ;---, \mathrm{pH} ;-\ldots$, growth.

strains. Other results are shown in Table II. Micromonospora strains utilized acidic, basic or acidic amide amino acids more extensively than neutral amino acids. The only exception to this was L-serine, which supported excellent growth of almost all the strains. L-Alanine $(\alpha-$ aminopropionic acid) and L-cysteine ( $\alpha$-amino$\beta$-mercaptopropionic acid) did not permit as much growth as L-serine ( $\alpha$-amino- $\hat{p}$ hydroxypropionic acid). Examination of the hydroxy- $\alpha$-aminobutyric acids showed Lthreonine, with its hydroxy group at the $\beta$ position, was utilized better than Lhomoserine, with it at the $\gamma$-position. Thus the $\beta$-hydroxy group seems to play a significant role in the utilization of neutral amino acids. In the Micromonospora strains used, L-alanine, L-histidine and L-lysine followed characteristic metabolic patterns.

The changes in growth rates and $\mathrm{pH}$ values during the incubation of the submerged cultures were examined using strains able to grow on the different nitrogen sources. Representive results are given in Fig. 3. L-Glutamic acid was an excellent nitrogen source for these strains at their optimum growth levels and rates. LAlanine yielded almost the same amounts of mycelial mass as ammonium sulfate or ammonium nitrate at 6 th day, but gave lower values at earlier stages, thus suggesting delayed responses of the organisms to the amino acid. Consumption of L-glutamic acid and sodium nitrate made the culture broth alkaline, whereas metabolism of L-lysine, L-alanine, ammonium sulfate and ammonium nitrate rendered it acidic.

\section{Carbon utilization}

A comparative study of the utilization of 24 carbohydrates was made. In both a defined agar medium and Leudemann's medium, abundant growth was attained with such sugars as glucose, maltose, sucrose and starch by all the strains used. However, little or no 
growth was attained when they were grown on dulcitol, sorbitol, L-sorbose or melezitose. Inositol permitted moderate growth of $M$. inositola ATCC 31010 on Luedemann's medium and poor growth on the defined medium. As shown in Table III, the degree of carbohydrate utilization in the defined medium did not coincide with that in Luedemann's medium. Generally, using the same carbohydrate, better utilization was observed with the Luedemann's medium rather than with the defined medium. This was noted especially in the case of Dfructose. On the other hand, a preference for raffinose and salicin utilization was observed with the defined medium. In addition, the consumption of lactose appeared to depend on the strain used rather than on the basal medium.

The utilization of raffinose, $\alpha$-melibiose, mannitol and L-rhamnose have been used as criteria in the classification of the species of Micromonospora (8th Bergey's Manual). In addition, other diagnostic utilization patterns were observed with D-arabinose, D-ribose, glycerol, cellobiose and salicin (Table III).

\section{Glycosidase activities}

Since glycosidase reactions are involved in metabolism of oligosaccharides, the ability to utilize the sugars is closely related to the presence of the enzymes. When tested with each of the $p$-nitrophenylglycosides, the activities of $\alpha$-glucosidase, $\beta$-glucosidase, $\beta$-galactosidase and $\beta$ - $N$-acetylglucosaminidase were detected in all the strains used, but those of $\alpha$ xylosidase, $\beta$-glucuronidase, $\alpha$-fucosidase and $\beta$-fucosidase were not (Table IV). Additional characteristics were found in other glycosidase activities. For example, no $\alpha$-galactosidase activity was detected in 9 strains, the spores of which are known to be bluntly spiny-surfaced or aberrant. No detectable $\beta$-xylosidase activity was found in 3 of 14 strains possessing $\alpha$ galactosidase activity. $\alpha$-Mannosidase activity was positive in only 3 strains.

A relationship between $\alpha$-glucosidase activity and the utilization of sucrose and maltose was observed in all the strains tested.
Other results obtained by comparing these 2 tests were not always consistent with one another. These discrepancies may be ascribed either to the use of $p$-nitrophenylglycosides as substrates for glycosidases or to the permeability of the glycosides.

All the positive reactions in this experiment were observed within $2 \mathrm{hr}$. Other experiments revealed that intact cells, collected either from an agar medium or from a submerged culture, gave almost the same results as sonicates of the cells.

\section{DISCUSSION}

In this study a chemically defined basal medium was devised to determine carbohydrate utilization by Micromonospora strains. The tests were clear, easy to perform and generally very reproducible. However, the results did not always correspond to those obtained using Luedemann's medium, which has been proposed to characterize the species of Micromonospora. ${ }^{6)}$ Since the presence of $0.5 \%$ yeast extract permitted appreciable growth of the strains on Luedemann's medium without carbohydrate, the degree of utilization might be difficult to determine in some cases. In fact, the results were inaccurate when the carbohydrates were poorly utilized or not utilized at all. This was often observed with D-galactose, Dribose and lactose. Another discrepancy was found using D-fructose, raffinose and salicin, since their metabolism was substantially affected by the kind of basal medium used. Others have reported similar results using just a few strains of Micromonospora. ${ }^{8 \sim 10)}$ With their strains, as well as ours, D-fructose allowed abundant growth on Luedemann's medium, but little or no growth on the chemically defined media.

A variety of glycosidase activities have been extensively investigated in bacteria. Recently the distribution of these enzymes in Actinomycetaceae and related organisms (nonspore-forming actinomycetes) was reported. ${ }^{11)}$ However, this information is not available for other genera of Actinomycetales, except for a 
Table III. Utilization of Carbohydrates by Micromonospora Strains Grown on Leudemann's Medium and a Defined Medium

\begin{tabular}{|c|c|c|c|c|c|c|c|c|c|c|c|c|c|c|c|c|c|}
\hline \multirow{3}{*}{ Micromonospora strains } & & \multicolumn{16}{|c|}{ Growth $^{a}$} \\
\hline & & \multicolumn{2}{|c|}{ D-Xylose } & \multicolumn{2}{|c|}{ Cellobiose } & \multicolumn{2}{|c|}{ D-Mannose } & \multicolumn{2}{|c|}{ Trehalose } & \multicolumn{2}{|c|}{ D-Fructose } & \multicolumn{2}{|c|}{ D-Galactose } & \multicolumn{2}{|c|}{ Lactose } & \multicolumn{2}{|c|}{ Raffinose } \\
\hline & & $\mathrm{L}^{b}$ & $\mathrm{C}^{c}$ & L & $\mathrm{C}$ & $\mathrm{L}$ & $\mathrm{C}$ & L & $\mathrm{C}$ & $\mathrm{L}$ & $\mathrm{C}$ & $\mathrm{L}$ & $\mathrm{C}$ & $\mathbf{L}$ & $\mathrm{C}$ & $\mathrm{L}$ & $\mathrm{C}$ \\
\hline M. coerulea & ATCC 27008 & ++ & ++ & ++ & ++ & ++ & ++ & + & \pm & ++ & ++ & ++ & ++ & \pm & + & ++ & ++ \\
\hline M. inositola & ATCC 31010 & ++ & ++ & ++ & ++ & ++ & ++ & ++ & ++ & ++ & ++ & ++ & ++ & ++ & ++ & + & ++ \\
\hline M. melanosporea & IFO 12515 & ++ & ++ & ++ & ++ & ++ & ++ & ++ & + & + & \pm & ++ & ++ & + & + & \pm & ++ \\
\hline M. purpureochromogenes & ATCC 27007 & ++ & ++ & ++ & ++ & ++ & ++ & + & + & ++ & + & ++ & ++ & ++ & + & ++ & ++ \\
\hline M. globosa & KCC A-0126 & ++ & ++ & ++ & ++ & ++ & ++ & ++ & ++ & ++ & + & ++ & ++ & ++ & ++ & ++ & ++ \\
\hline M. fusca & NRRL B-943 & ++ & ++ & ++ & ++ & ++ & + & + & + & ++ & \pm & ++ & + & ++ & ++ & ++ & ++ \\
\hline M. chalcea & ATCC 12452 & ++ & ++ & ++ & ++ & ++ & ++ & ++ & + & ++ & $\bar{t}$ & ++ & ++ & ++ & + & ++ & ++ \\
\hline M. narashino & KCC A-0129 & ++ & ++ & ++ & ++ & ++ & ++ & ++ & ++ & ++ & + & ++ & ++ & \pm & \pm & + & ++ \\
\hline M. halophytica subsp. nigra & NRRL 3097 & ++ & ++ & ++ & ++ & ++ & ++ & + & \pm & ++ & \pm & ++ & ++ & ++ & ++ & \pm & + \\
\hline M. carbonacea & NRRL 2972 & ++ & ++ & ++ & ++ & ++ & ++ & ++ & ++ & ++ & \pm & ++ & ++ & ++ & ++ & + & ++ \\
\hline M. carbonacea subsp. aurantica & NRRL 2997 & ++ & ++ & ++ & ++ & ++ & ++ & ++ & ++ & \pm & - & ++ & ++ & + & ++ & + & ++ \\
\hline$M \cdot$ parva & KCC A-0127 & ++ & ++ & ++ & ++ & ++ & ++ & ++ & ++ & ++ & + & ++ & ++ & ++ & ++ & + & ++ \\
\hline M. rosaria & NRRL 3718 & ++ & ++ & ++ & ++ & ++ & ++ & ++ & + & ++ & - & ++ & ++ & + & + & - & - \\
\hline M. megalomicea subsp. nigra & NRRL 3275 & ++ & ++ & ++ & + & ++ & + & + & + & ++ & \pm & \pm & \pm & \pm & - & - & - \\
\hline M. grisea & NRRL 3800 & ++ & ++ & ++ & ++ & ++ & ++ & ++ & ++ & ++ & \pm & ++ & ++ & \pm & - & - & - \\
\hline M. olivasterospora & ATCC 21819 & ++ & ++ & + & + & ++ & ++ & ++ & ++ & ++ & ++ & ++ & ++ & - & - & - & - \\
\hline M. echinospora subsp. pallida & NRRL 2996 & + & + & ++ & ++ & ++ & ++ & ++ & ++ & ++ & ++ & + & \pm & - & - & - & - \\
\hline M. zionensis & NRRL 5466 & ++ & ++ & ++ & ++ & ++ & ++ & ++ & ++ & + & + & ++ & ++ & - & - & - & - \\
\hline M. purpurea & NRRL 2953 & ++ & ++ & ++ & ++ & ++ & ++ & ++ & ++ & + & \pm & + & + & \pm & - & - & - \\
\hline M. echinospora & NRRL 2985 & ++ & ++ & ++ & ++ & ++ & ++ & ++ & ++ & \pm & - & + & - & $\overline{ \pm}$ & - & - & - \\
\hline M. echinospora subsp. ferruginea & NRRL 2995 & ++ & ++ & ++ & ++ & ++ & ++ & ++ & ++ & \pm & - & \pm & - & \pm & - & - & - \\
\hline M. sagamiensis & ATCC 21803 & ++ & ++ & ++ & + & ++ & + & ++ & ++ & ++ & \pm & ++ & ++ & ++ & + & - & - \\
\hline M. inyoensis & NRRL 3292 & + & \pm & - & - & ++ & ++ & ++ & ++ & + & - & ++ & ++ & + & \pm & - & - \\
\hline
\end{tabular}


TABLE III. (continued)

\begin{tabular}{|c|c|c|c|c|c|c|c|c|c|c|c|c|c|c|c|}
\hline \multirow{3}{*}{ Micromonospora strains } & & \multicolumn{14}{|c|}{ Growth $^{a}$} \\
\hline & & \multicolumn{2}{|c|}{ Salicin } & \multicolumn{2}{|c|}{$\alpha$-Melibiose } & \multicolumn{2}{|c|}{ Mannitol } & \multicolumn{2}{|c|}{ Glycerol } & \multicolumn{2}{|c|}{ L-Rhamnose } & \multicolumn{2}{|c|}{ D-Arabinose } & \multicolumn{2}{|c|}{ D-Ribose } \\
\hline & & $\mathrm{L}^{b}$ & $\mathrm{C}^{c}$ & L & $\mathrm{C}$ & $\mathrm{L}$ & $\mathrm{C}$ & $\mathrm{L}$ & $\mathrm{C}$ & $\mathrm{L}$ & $\mathrm{C}$ & L & $\mathrm{C}$ & $\mathrm{L}$ & $\mathrm{C}$ \\
\hline M. coerulea & ATCC 27008 & - & - & ++ & ++ & - & - & - & - & - & - & - & - & - & - \\
\hline M. inositola & ATCC 31010 & - & - & ++ & ++ & - & - & - & - & - & - & - & - & - & - \\
\hline M. melanosporea & IFO 12515 & - & - & ++ & ++ & - & - & - & - & - & - & - & - & - & - \\
\hline M. purpureochromogenes & ATCC 27007 & - & - & ++ & ++ & - & - & - & - & - & - & - & - & - & - \\
\hline M. globosa & KCC A-0126 & \pm & \pm & ++ & ++ & - & - & - & - & - & - & - & - & - & - \\
\hline M. fusca & NRRL B-943 & - & \pm & ++ & ++ & - & - & - & - & - & - & - & - & - & - \\
\hline M. chalcea & ATCC 12452 & - & \pm & ++ & ++ & - & - & - & - & - & - & - & - & - & - \\
\hline M. narashino & KCC A-0129 & - & $\overline{ \pm}$ & + & ++ & - & - & - & - & - & - & - & - & - & - \\
\hline M. halophytica subsp. nigra & NRRL 3097 & \pm & + & + & + & - & - & - & - & - & - & - & - & - & - \\
\hline M. carbonacea & NRRL 2972 & \pm & + & ++ & ++ & - & - & ++ & ++ & - & - & - & - & - & - \\
\hline M. carbonacea subsp. aurantica & NRRL 2997 & \pm & + & ++ & ++ & ++ & ++ & - & - & - & - & - & - & - & - \\
\hline$M$. parva & KCC A-0127 & \pm & ++ & ++ & ++ & ++ & ++ & - & - & - & - & - & - & - & - \\
\hline M. rosaria & NRRL 3718 & \pm & ++ & \pm & \pm & ++ & + & - & - & ++ & ++ & ++ & ++ & \pm & \pm \\
\hline M. megalomicea subsp. nigra & NRRL 3275 & - & - & + & \pm & - & - & - & - & - & - & - & - & - & - \\
\hline M. grisea & NRRL 3800 & - & - & - & - & - & - & - & - & \pm & \pm & + & \pm & \pm & + \\
\hline M. olivasterospora & ATCC 21819 & - & - & - & - & - & - & - & - & ++ & ++ & + & \pm & - & - \\
\hline M. echinospora subsp. pallida & NRRL 2996 & - & - & - & - & - & - & - & - & ++ & ++ & + & $\overline{ \pm}$ & + & + \\
\hline M. zionensis & NRRL 5466 & - & - & - & - & - & - & - & - & ++ & ++ & + & ++ & \pm & - \\
\hline M. purpurea & NRRL 2953 & - & - & - & - & - & - & - & - & - & - & ++ & ++ & \pm & - \\
\hline M. echinospora & NRRL 2985 & - & - & - & - & - & - & - & - & - & - & ++ & + & ++ & ++ \\
\hline M. echinospora subsp. ferruginea & NRRL 2995 & - & - & - & - & - & - & - & - & - & - & - & - & ++ & ++ \\
\hline M. sagamiensis & ATCC 21803 & - & - & - & - & - & - & - & - & - & - & - & \pm & - & - \\
\hline M. inyoensis & NRRL 3292 & - & - & - & - & - & - & - & - & - & - & - & - & - & - \\
\hline
\end{tabular}

a Growth after incubation for 2 weeks at $28^{\circ} \mathrm{C}(++$, abundant; + , moderate; \pm , poor; -, no growth $)$.

$b \quad$ Luedemann's medium $\left(1 \%\right.$ carbohydrate $+0.5 \%$ yeast extract $\left.+0.1 \% \mathrm{CaCO}_{3}\right)$.

Relative growth to that on the medium containing glucose $(++)$ and no carbohydrate $(-)$.

c A chemically defined medium ( $1 \%$ carbohydrate + basal medium). 
Table IV. Glycosidase Activities of Micromonospora Strains Using $p$-Nitrophenylglycosides as Substrates

Activity $^{a}$

\begin{tabular}{|c|c|c|c|c|c|c|c|c|c|c|c|c|}
\hline Micromonospora strains & & 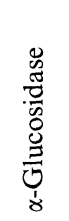 & 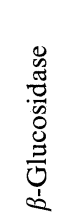 & 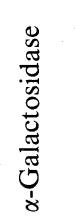 & 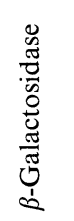 & 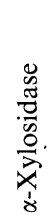 & 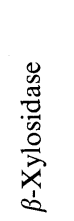 & 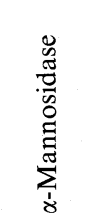 & 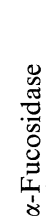 & 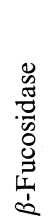 & 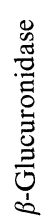 & 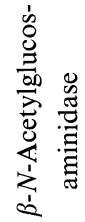 \\
\hline M. chalcea & ATCC 12452 & ++ & ++ & ++ & ++ & - & ++ & - & - & - & - & ++ \\
\hline M. halophytica subsp. nigra & NRRL 3097 & ++ & ++ & ++ & ++ & - & ++ & - & - & - & - & ++ \\
\hline M. carbonacea & NRRL 2972 & ++ & ++ & ++ & ++ & - & ++ & - & - & - & - & ++ \\
\hline M. carbonacea subsp. aurantica & NRRL 2997 & ++ & ++ & ++ & ++ & - & ++ & - & - & - & - & \pm \\
\hline M. melanosporea & IFO 12515 & ++ & ++ & ++ & ++ & - & ++ & - & - & - & - & ++ \\
\hline M. globosa & KCC A-0126 & ++ & ++ & ++ & ++ & - & ++ & - & - & - & - & ++ \\
\hline M. parva & KCC A-0127 & ++ & ++ & ++ & ++ & - & ++ & - & - & - & - & ++ \\
\hline M. megalomicea subsp. nigra & NRRL 3275 & ++ & ++ & ++ & ++ & - & ++ & - & - & - & - & ++ \\
\hline M. fusca & NRRL B-943 & ++ & ++ & ++ & ++ & - & ++ & - & - & - & - & ++ \\
\hline M. narashino & KCC A-0129 & ++ & ++ & ++ & ++ & - & - & - & - & - & - & ++ \\
\hline M. purpureochromogenes & ATCC 27007 & ++ & ++ & ++ & ++ & - & - & - & - & - & - & ++ \\
\hline M. coerulea & ATCC 27008 & ++ & ++ & ++ & ++ & - & - & ++ & - & - & - & ++ \\
\hline M. inositola & ATCC 31010 & ++ & ++ & ++ & ++ & - & ++ & ++ & - & - & - & ++ \\
\hline M. rosaria & NRRL 3718 & ++ & ++ & ++ & ++ & - & ++ & + & - & - & - & ++ \\
\hline M. purpurea & NRRL 2953 & ++ & ++ & - & ++ & - & ++ & - & - & - & - & ++ \\
\hline M. sagamiensis & ATCC 31083 & ++ & ++ & - & ++ & - & ++ & - & - & - & - & ++ \\
\hline M. echinospora subsp. pallida & NRRL 2996 & ++ & ++ & - & ++ & - & ++ & - & - & - & - & ++ \\
\hline M. echinospora subsp. ferruginea & NRRL 2995 & ++ & ++ & - & ++ & - & \pm & - & - & - & - & ++ \\
\hline M. echinospora & NRRL 2985 & ++ & ++ & - & ++ & - & \pm & - & - & - & - & ++ \\
\hline M. zionensis & NRRL 5466 & ++ & ++ & - & ++ & - & - & \pm & - & - & - & ++ \\
\hline M. grisea & NRRK 3800 & ++ & ++ & - & ++ & - & - & - & - & - & - & ++ \\
\hline M. olivasterospora & ATCC 21819 & ++ & ++ & - & ++ & - & - & - & - & - & - & ++ \\
\hline$M$. inyoensis & NRRL 3292 & ++ & ++ & - & ++ & - & - & - & - & - & - & ++ \\
\hline
\end{tabular}

${ }^{a}++$, strongly positive; + , positive; \pm , very weak; - , negative. 
Table V. Characteristics of Micromonospora Species and Strains

\begin{tabular}{|c|c|c|c|c|c|c|c|c|c|c|c|c|c|c|c|c|c|c|}
\hline \multirow[b]{3}{*}{ Micromonospora strains } & & \multicolumn{3}{|c|}{ Glycosidase } & \multicolumn{10}{|c|}{ Utilization } & \multirow{3}{*}{ 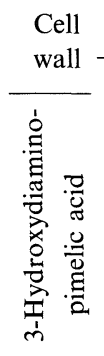 } & \multirow{3}{*}{$\begin{array}{c}\text { Spore } \\
\end{array}$} & \multicolumn{2}{|c|}{$\begin{array}{c}\text { Diagnostic } \\
\text { Pigment }\end{array}$} \\
\hline & & \multirow[b]{2}{*}{ 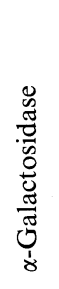 } & \multirow[b]{2}{*}{ 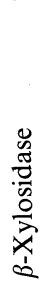 } & \multirow[b]{2}{*}{ 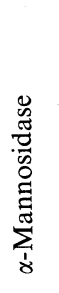 } & \multirow[b]{2}{*}{ 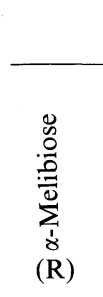 } & \multirow[b]{2}{*}{ 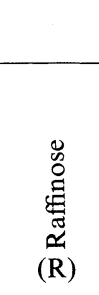 } & \multicolumn{5}{|c|}{ Carbohydrate } & \multicolumn{3}{|c|}{ Amino acid } & & & & \\
\hline & & & & & & & 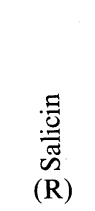 & 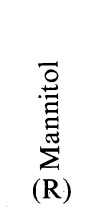 & 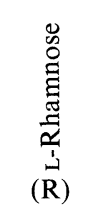 & 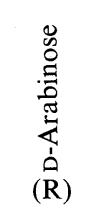 & 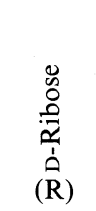 & 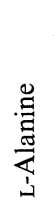 & 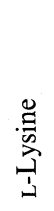 & 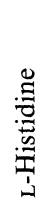 & & & $\begin{array}{l}\frac{\pi}{\mathbb{J}} \\
\sum \\
\sum\end{array}$ & $\begin{array}{l}\frac{0}{0} \\
\frac{3}{0} \\
\infty\end{array}$ \\
\hline M. globosa & KCC A-0126 & + & + & - & $+(\mathrm{n})$ & $+(\mathrm{n})$ & $\pm(n)$ & $-(n)$ & $-(\mathrm{n})$ & $-(n)$ & $-(\mathrm{n})$ & + & + & + & - & - & & \\
\hline M. fusca & NRRL B-943 & + & + & - & $+(+)$ & $+(-)$ & $\pm(-)$ & $-(+)$ & $-(-)$ & $-(-)$ & $-( \pm)$ & + & \pm & + & - & - & & $\mathrm{Br}$ \\
\hline M. chalcea & ATCC 12452 & + & + & - & $+(+)$ & $+(+)$ & $\pm(-)$ & $-(-)$ & $-(-)$ & $-(-)$ & $-(-)$ & + & - & + & - & - & & \\
\hline M. melanosporea & IFO 12515 & + & + & - & $+(\mathrm{n})$ & $+(\mathrm{n})$ & $-(\mathrm{n})$ & $-(\mathrm{n})$ & $-(\mathrm{n})$ & $-(\mathrm{n})$ & $-(n)$ & + & - & + & - & - & & \\
\hline M. megalomicea subsp. nigra & NRRL 3275 & + & + & - & $\pm(-)$ & $-(-)$ & $-(\mathrm{n})$ & $-(-)$ & $-(-)$ & $-(-)$ & $-(-)$ & - & - & \pm & + & - & & \\
\hline M. carbonacea & NRRL 2972 & + & + & - & $+(+)$ & $+( \pm)$ & $+(-)$ & $-(-)$ & $-(-)$ & $-(-)$ & $-(-)$ & - & + & $\overline{+}$ & + & - & & \\
\hline M. carbonacea subsp. autantica & NRRL 2997 & + & + & - & $+(+)$ & $+( \pm)$ & $+(-)$ & $-(-)$ & $-(-)$ & $-(-)$ & $-(-)$ & \pm & + & + & + & - & & \\
\hline M. halophytica subsp. nigra & NRRL 3097 & + & + & - & $+(+)$ & $+( \pm)$ & $-(\mathrm{n})$ & $-(-)$ & $-(-)$ & $-(-)$ & $-(-)$ & - & \pm & + & + & - & & \\
\hline$M$. parva & KCC A-0127 & + & + & - & $+(\mathrm{n})$ & $+(-)$ & $+(\mathrm{n})$ & $-(-)$ & $-(-)$ & $-(n)$ & $-(n)$ & + & \pm & - & + & - & & \\
\hline M. rosaria & NRRL 3718 & + & + & + & $\pm( \pm)$ & $-(-)$ & $+(-)$ & $+(+)$ & $+(+)$ & $+(+)$ & $\pm(+)$ & + & + & + & + & - & & W-R \\
\hline M. inositola & ATCC 31010 & + & + & + & + & + & - & + & - & - & - & + & + & + & + & - & & \\
\hline M. coerulea & ATCC 27008 & + & - & + & $+(+)$ & $+(+)$ & $-(\mathrm{n})$ & $+(+)$ & $-(-)$ & $-(-)$ & $-(-)$ & + & + & + & - & - & $\mathrm{B}-\mathrm{Gr}$ & \\
\hline M. narashino & KCC A-0129 & + & - & - & $+(\mathrm{n})$ & $+(n)$ & $\pm(n)$ & $-(n)$ & $-(\mathrm{n})$ & $-(\mathrm{n})$ & $-(\mathrm{n})$ & + & + & + & - & - & & \\
\hline M. purpureochromogenes & ATCC 27007 & + & - & - & $+(+)$ & $+(+)$ & $-(\mathrm{n})$ & $-(-)$ & $-(-)$ & $-(-)$ & $-(-)$ & + & \pm & - & - & - & & \\
\hline M. echinospora & NRRL 2985 & - & \pm & - & $-(-)$ & $-(-)$ & $-(-)$ & $-(-)$ & $+(+)$ & $\pm(-)$ & $-(-)$ & + & + & + & + & + & $\mathrm{Pu}$ & \\
\hline M. echinospora subsp. ferruginea & NRRL 2995 & - & \pm & - & $-(-)$ & $-(-)$ & $-(-)$ & $-(-)$ & $+(+)$ & $\pm(-)$ & $+(+)$ & \pm & + & + & + & + & $\mathrm{Pu}$ & \\
\hline M. echinospora subsp. pallida & NRRL 2996 & - & $\overline{+}$ & - & $-(-)$ & $-(-)$ & $-(-)$ & $-(-)$ & $+(+)$ & $+(-)$ & $-( \pm)$ & $\bar{t}$ & + & + & + & + & & \\
\hline$M$. purpurea & NRRL 2953 & - & + & - & $-(-)$ & $-(-)$ & $-(-)$ & $-(-)$ & $+(-)$ & $\pm(-)$ & $+(+)$ & + & + & + & + & $\mathrm{n}$ & $\mathrm{Pu}$ & \\
\hline M. sagamiensis & ATCC 21803 & - & + & - & - & - & - & - & - & + & - & - & \pm & + & + & + & & $\mathrm{Br}$ \\
\hline M. grisea & NRRL 3800 & - & - & - & $-(-)$ & $-(-)$ & $-(-)$ & $-(-)$ & $-(-)$ & $+(+)$ & $+(\mathrm{n})$ & \pm & - & + & + & + & & \\
\hline M. olivasterospora & ATCC 21819 & - & - & - & - & - & - & - & - & - & + & - & \pm & + & + & + & & \\
\hline M. zionensis & NRRL 5466 & - & - & \pm & $-(-)$ & $-(-)$ & $-(-)$ & $-(-)$ & $-(-)$ & $+(+)$ & $-(n)$ & + & - & + & + & + & & \\
\hline M. inyoensis & NRRL 3292 & - & - & - & $-(-)$ & $-(-)$ & $-(\mathrm{n})$ & $-(-)$ & $-(-)$ & $-(-)$ & $-(+)$ & - & \pm & + & + & + & & \\
\hline
\end{tabular}

$\mathrm{Br}$, brown; W-R, wine-red; B-Gr, blue-green; $\mathrm{Pu}$, purple; $\mathrm{R}$, data in the description of each strain; $\mathrm{n}$, not reported;,+ positive; \pm , weakly positive; - , negative. 
few enzymes in Streptomyces. ${ }^{12 \sim 15)}$ Of all the glycosidases tested, neither $\alpha$-fucosidase nor $\beta$ glucuronidase activity was found in Micromonospora strains. The former has been demonstrated in the strains of Actinomyces, ${ }^{11)}$ Clostridium, ${ }^{16)}$ and Streptococcus,${ }^{17,18)}$ whereas the latter has been found in the strains of Bifidobacterium, ${ }^{11)} \quad$ Propionibacterium, ${ }^{11}$ ) Streptococcus, ${ }^{19 \sim 21)} \quad$ Escherichia $^{7)}$ and Shigella. ${ }^{7)}$ However, $\beta$ - $N$-acetylglucosaminidase activity was detected in all these species, but was almost undetectable in the strains of Actinomycetaceae. Thus, Micromonospora species have a different pattern of glycosidase activities from the organisms previously described.

The activities of glycosidases may be induced by the presence of potential substrate, such as lactose for $\beta$-galactosidase. ${ }^{22,23)}$ However, it is conceivable that a reasonable comparison of these activities can be made if the organisms are cultivated and tested under prescribed conditions. Such a test allows a simple and rapid detection, and the substrates used are commercially available. Since most Micromonospora strains required a long incubation time and special conditions for conventional testing their biological properties, we believe that this test can provide a useful mean of recognition in the classification of Micromonospora species.

Since the morphological properties and pigments in Micromonospora are not so diverse as they are in Streptomyces, then the biological and physiological characteristics of the species play an important role in their classification. In the 8th Edition of Bergey's Manual, the following identification criteria are used: carbohydrate utilization with $\alpha$-melibiose, raffinose, L-rhamnose and mannitol, hydrolysis of cellulose and growth on Czapeck's sucrose agar or protein-containing agar. Unfortunately, the insufficient descriptions of the species proposed by early workers make their classification a difficult one. However, this study demonstrated additional characteristics of the strains which are summarized in Table $\mathrm{V}$ along with other properties, such as the presence of 3-hydroxydiaminopimelic acid in the cell walls, ${ }^{3)}$ bluntly spiny-surfaced spores, and diagnostic pigments. Among them, two of the properties - $\alpha$-galactosidase and bluntly spiny-surfaced spores - occurred simultaneously. The strains possessing $\alpha$ galactosidase activity can be divided into 5 groups according to the presence or absence of $\beta$-xylosidase activity, $\alpha$-mannosidase activity and 3-hydroxydiaminopimelic acid. The first group, including $M$. globosa KCC A0126, M. fusca NRRL B-943, M. chalcea ATCC 12452 and M. melanosporea IFO 12515, demonstrated $\beta$-xylosidase activity but no $\alpha$-mannosidase activity, and did not contain 3-hydroxydiaminopimelic acid. In this group, the species (strains) were differentiated from each other by their utilization of $\mathrm{L}$ tryptophan (Table II) and L-lysine, and by the presence of soluble pigments. The second group, differing from the first in the presence of 3-hydroxydiaminopimelic acid, included $M$. megalomicea subsp. nigra NRRL 3275, M. carbonacea NRRL 2972, M. carbonacea subsp. aurantica NRRL 2997 and $M$. halophytica subsp. nigra NRRL 3097. These species (strains) also apparently have different abilities to utilize raffinose, salicin, L-alanine, L-lysine and L-histidine. The third group is characterized by positive responses to all of the above-mentioned determinants. $M$. inositola ATCC 31010 and $M$. rosaria NRRL 3718 belong to this group, and are clearly separated by their utilization of raffinose, salicin, L-rhamnose and Darabinose and the presence of soluble pigment. The fourth group contains $M$. coerulea ATCC 27008, which has $\alpha$-mannosidase activity, but lacks $\beta$-xylosidase activity and 3hydroxydiaminopimelic acid. The fifth group is distinct from the other four in that it lacks two enzyme activities as well as 3-hydroxydiaminopimelic acid. In this group, $M$. narashino KCC A-0129 differs from $M$. purpureochromogenes ATCC 27007 in its utilization of L-histidine. In addition, the strains lacking $\alpha$-galactosidase (the sixth group), most of which are known to produce characteristic 
pigments, show differences in $\beta$-xylosidase activity and the metabolism of L-rhamnose, Darabinose, D-ribose, L-alanine and L-lysine. Thus, we belive that comparison of the properties shown in Table $\mathrm{V}$ will contribute to a more precise identification of Micromonospora species.

The mycelial yields of the strains tested were significantly reduced by concentrations of phosphate higher than $0.01 \mathrm{M}$. Their doubling times were found to be 2 to 4 times longer than those reported for Streptomyces. ${ }^{24)}$ Moreover, a vitamin requirement for growth was found in some strains. These properties, along with their susceptibility to acidic conditions and lessened tolerance to sodium chloride already described, ${ }^{25,26)}$ might also explain the rare occurrence of Micromonospora in soil.

Acknowledgments. The authors thank Dr. A. Seino of the Central Research Laboratories, Kaken Chemical Co., Tokyo (Japan), for the gift of many strains of Micromonospora.

\section{REFERENCES}

1) I. Kawamoto, The Actinomycetalogist, 35, 2 (1979).

2) G. H. Wagman and M. J. Weinstein, Ann. Rev. Microbiol., 34, 537 (1980).

3) I. Kawamoto, T. Oka and T. Nara, J. Bacteriol., 146, 527 (1981).

4) I. Kawamoto, T. Oka and T. Nara, Agric. Biol. Chem., 46, 221 (1982).

5) T. G. Pridham and D. Gottlieb, J. Bacteriol., 56, 107 (1948).

6) G. M. Luedemann and B. C. Brodsky, Antimicrob.
Agents \& Chemother.-1963, 116 (1964).

7) M. Kilian and P. Bülow, Acta Path. Microbiol. Scand. Sect. B, 84, 245 (1976).

8) K. Hatano, E. Higashide and M. Shibata, J. Antibiot., 29, 1166 (1976).

9) H. Maehr, C. Liu, T. Herman, B. T. Prosser, J. M. Smallheer and N. J. Palleroni, J. Antibiot., 33, 1431 (1980).

10) Y. Kawamura, Y. Yasuda and M. Mayama, J. Antibiot., 34, 367 (1981).

11) M. Kilian, J. Clin. Microbiol., 8, 127 (1978).

12) A. J. Lyons, T. G. Pridham and C. W. Hesseline, Appl. Microbiol., 19, 579 (1969).

13) A. Dan and G. Szabo, Acta Biol. Acad. Sci. Hung., 24, 1 (1973).

14) J. Sanchez and C. Hardisson. Can. J. Microbiol., 25, 833 (1979).

15) S. Challerjee and L. C. Vining, Can. J. Microbiol., 27, 639 (1981).

16) D. Aminoff and K. Furukawa, J. Biol. Chem., 245, 1659 (1970).

17) C. E. Nord and T. Wadström, Med. Microbiol. Immunol., 158, 95 (1972).

18) M. Kilian, J. Gen. Microbiol., 93, 9 (1976).

19) D. E. O. Willias J. Gen. Microbiol., 10, 337 (1954).

20) C. E. Nord, L. Linder, T. Wadström and A. A. Lindberg, Acta Oral Biol., 18, 391 (1973).

21) T. O. Röd, R. H. Haug and T. Midtvedt, Acta Pathol. Microbiol. Scand. Sect. B, 82, 533 (1974).

22) J. Sanchez and C. Hardisson, Curr. Microbiol., 4, 91 (1981).

23) J. Sanchez, M. F. Aias and C. Hardisson, Experientia, 37, 469 (1981).

24) T. H. Flowers and S. T. Williams, J. Gen. Microbiol., 98, 285 (1977).

25) G. M. Luedemann, Adv. Appl. Microbiol., 11, 101 (1970).

26) G. M. Luedemann, Trans. N. Y. Acad. Sci., 33, 207 (1971). 